\title{
Design and Development of Electric Wheelchair for differently abled person
}

\author{
Ankit Kumar Mishra $^{\# 1}$, Krishna K. Warhade ${ }^{* 2}$ \\ ${ }^{*}$ M.E. student at Department of Electronics \& Telecommunication, MIT College of Engineering \\ Pune, India \\ 1 ankitmishra150393@gmail.com \\ * Professor at Department of Electronics \& Telecommunication, MIT College of Engineering \\ Pune, India \\ ${ }^{2}$ krishna.warhade@mitcoe.edu.in
}

\begin{abstract}
This paper proposed development of Electric wheelchair for Brain Computing Interface(BCI) system to make contribution in biomedical field, these devices assist people who are physically handicapped, paraplegic patients can easily use the electric wheelchair using joystick or by some other interfaces but there is segment of people who suffer from diseases like motor neuron disease (MND) and they can not operate the wheelchair using interfaces like joystick, such people can only drive the wheelchair using eye blinking or brain signals. In some cases, electric wheelchair can be operated using BCI (Brain Computing Interface) and providing absolute control to such wheelchair is important, configuration of these wheelchair is discussed in this proposed system. Utilizing various resources to build cost efficient system, how sensors and drivers are being interfaced with the Microcontroller and their working to drive the wheelchair according to the acquired signal by brain.
\end{abstract}

Keyword-Ultrasonic sensor, Dual H bridge, Electroencephalography (EEG), Brain Computer Interface (BCI)

\section{INTRODUCTION}

Electric Wheelchairs assisting the elderly as well as the differently abled person and considered as one of the most important mobility aids available commercially. While the needs of many people with disabilities can be fulfilled with electric wheelchairs using joystick or keys interface, but still there are number of people who are fully paralyzed and they can move neither upper body nor lower body. To address such problems and assisting the people who are suffering from this particular disability, Brain Computing Interface(BCI) system has been developed, it excludes the conventional ways of communication and establish a direct connection between the human brain and assisting devices.

The wheelchair is controlled by acquiring signals from the brain and brain computing interface is used with the controller to perform the desired action by the patient. In any electric wheelchair configuration, the components like motors, batteries, controller, type of wheels plays important role in design and development of electric wheelchair. Selection of reliable wheels, using efficient motors and batteries, designing the required controller in the wheelchair are important decision to take when developing electric wheelchair. Description of power wheelchair bases and frames, the advantages and potential limitations of different wheelchairs using by available drive wheel conf gurations. Additionally, various base options, seating options, and accessories are also considered as important factors to assist the patient in most appropriate way. There is no specific size or configuration that $\mathrm{fts}$ for all powered wheelchair and we can not conclude that this particular wheelchair is perfect for all because it totally depends on the requirement of individuals, all drive configuration have their advantages and disadvantages. It is necessary to have a clear understanding and knowledge of these pros and cons. The outcome of not focusing on these factors could be that patient will experience unnecessary limitations and discomfort in driving the wheelchair.

Three drive configurations are commonly popular in electric wheelchair as shown in Fig. 1 rear wheel drive, mid wheel drive and front wheel drive, Rear wheel drive have drive wheels located in the rear of the wheelchair or we can simply say motor is attached to the rear wheel, such configuration is capable of providing highest speeds but also having largest turning radius which reduces its use in indoor purposes. Mid wheel drive have wheels located in the center or in the middle of the wheelchair and hence, more weight is distributed centrally and has smallest turning radius which makes this configuration more suitable for indoor purposes. Front wheel drive have motor attached in the front wheel, these wheelchairs can easily bring on the obstacle and also capable in both indoor and outdoor purposes. As our concern is to drive the wheelchair by acquiring brain signals, we consider to have rear wheel drive configuration as more weight of the patient would be distributed in backwards. 
Basically, one has to select the wheelchair according to the needs of the patient. In designing wheelchair of any controlling option, few things should be kept in mind before selecting the materials we are going to use, starting from selection of wheels to cost efficient motors, everything should be good in terms of durability, machineability, reliability etc. These factors will cause irritation and frustration in driving the wheelchair to the patient even if electronics incorporation is perfectly done. In selection of wheels we prefer pneumatic drive wheels instead of solid insert drive wheels, as it was concluded by Andrew M. Kwarciak et al. pneumatic tyres exhibit lower rolling resistance as compared to solid tyres [1] and low rolling resistance tyres minimize wasted energy as tire rolls.
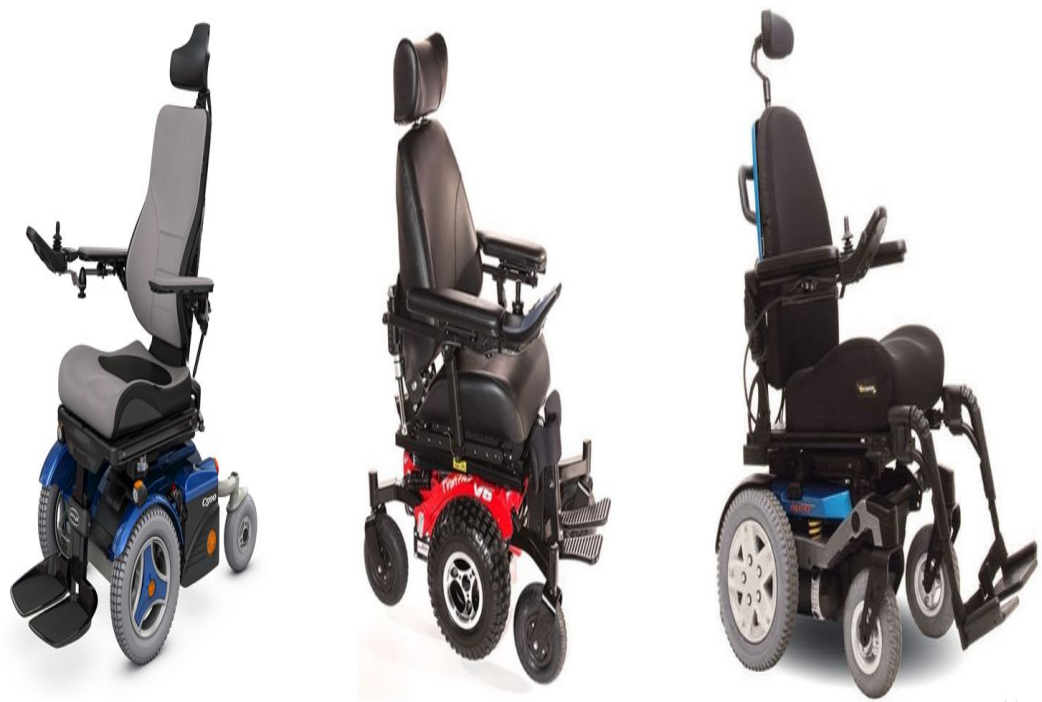

Fig. 1. Different drive wheel configurations [13]

There are several wheelchairs available commercially that gives people a better way of comfort ability. Some wheelchairs manufacturers provide portability to its consumer, various wheelchairs are available that can be port easily from one place to another. EZ Lite CRUISER is series of such existing portable wheelchair available in the market, one of its advantage include durable material and easy handling in shifting it. Patients have different heights and their need might be different at some particular point, therefore, seat adjustment also plays important role in providing the consumer a good comfort zone in driving the wheelchair. Wheelchair manufacturer Quickie sells wheelchair that has seat adjustment as well as different dynamic suspension techniques that gives consumer a better way to bring on the obstacles in the path but these wheelchair are available with very expensive price tag and not affordable for so many people comes into this disabled category.

\section{A. Collaboration with Brain Computing Interface}

Collaboration of brain computing interface includes a channel or medium through which signals can be transferred. EEG signals can be fed into the bluetooth module on the port of microcontroller UART port. The bluetooth module is basically used to transmit and receive the EEG signals in the system but the system is not limited to Bluetooth only, data or signals can also be transferred by using other modes wireless of communication. Zigbee module also can be used in several cases for data transfer between brain computer interface and wheelchair control.

\section{RELATED WORK}

Electric wheelchair previously used in so many paralysis cases where electric wheelchair operated using joystick, keyboard, voice, eye blinking etc. Electrooculography (EOG) signals also used in many researches, Electrooculography signals are used to record the eye movement and wheelchair is controlled by these eye movements, eye blink can be interpreted in various command to move wheelchair in forward and backward direction. In many studies, hands free control is also implemented and wheelchair is controlled using head movements.

Tom Carlson et al. have used commercially available Invacare wheelchair that is having mid-wheel drive but they modified it to get desired functionality. They have developed a remote joystick module that acts as an interface between laptop computer and wheelchair control network which is based on CANBUS and gives direct control from laptop to wheelchair, they have added wheel encoder to the drive wheels to get feedback to the wheelchair about its motion and array of solar sensors and webcams also added to wheelchair for providing 
environmental feedback to the controller of the wheelchair. To have visual feedback for the user, display is mounted and power distribution unit also used to supply sufficient power from batteries for all sensors, laptop and the display [2].

In some studies, Emotion Fractal Analysis Method (EFAM) is also considered as accurate method in BCI system. System that accurately recognizes and isolates emotions like delight, anger sorrow and pleasure using EFAM proposed by Nobuaki Kobayashi et al. and this system can quantify emotion based on the data captured in the form of electroencephalography. Wheelchair is controlled by using the data obtained in real time by EFAM [3].

Imran Ali Mirza et al. have designed mind controlled wheelchair using Arduino microcontroller which makes the movement of wheelchair [4]. In this proposed system, Neurosky Mindwave Headset is used and processed inputs from the BCI are received by Arduino microcontroller with the help of ThinkGear.net library. This Arduino based wheelchair is built using a servo motor which is a rotary actuator that provide control of angular position, velocity and acceleration.

Autonomous BCI wheelchair is also part of current researches, steady state visual evoked potential (SSVEP)based BCI is used to select the desired destination by Danny wee-kiat et al. [5]. Wheelchair navigation system is used which control the wheelchair autonomously by communicating according to the desired destination selected initially. Ultrasonic sensors are also used in the wheelchair to avoid the obstacles in the path, the number of $\mathrm{BCI}$ decisions needed to move the wheelchair to the desired destination is also reduced by transferring the responsibility of control to the navigation software. Path planning algorithm is used with predefined map where location information is obtained from laser and obstacle information obtained by ultrasonic sensors. Wheelchair will automatically detect a new path to the destination whenever obstacle is detected in the path and it will only stop when all paths are blocked and there is no obstacle-free path available to the destination.

B. Jenita Amali Rani et al. have designed robotic wheelchair module which consist of ARM 7 processor based on 32 bit operation with reduced instruction set computer (RISC) for controlling the movement of wheelchair by capturing the command received from BCI system module NeuroSky. The desired actions for movement of wheelchair is assigned on MATLAB by interrupt threshold, different interrupts are assigned for different operations like forward, backward, left, right, stop, rotate clockwise etc. ARM 7 processor is used for receiving signals from RF module by which motor is controlled and directed towards the desired movement of the wheelchair accordingly, KEIL software is used to compile the program and simulate the interrupt command to the DC motor. Here, RF transmitter module is connected to PC via serial data converter and processed values are sent via RS 232 to the Xbee receiver [6].

Wireless electroencephalogram (EEG) based brain computer interface (BCI) and a drive circuit that can drive DC motors of electric wheelchair which receives data through Bluetooth interface designed by Jzau-Sheng Lin et al. [7]. In this system, Microprocessor control unit SunPlus SPCE61A is used and DSP algorithm embedded for extracting the attention signal, they have used 4-set of ultrasonic module to have detection beside the left and right sides. Darlington circuits used as driving circuits to drive left and right motors, these amplify current to increase the torque of the motors and operation amplifier in motor driver adjust the speed of wheelchair. Attention signal is extracted from EEG signals that moves the wheelchair in forward direction but attention signal should be greater than the defined threshold and user can stop the wheelchair using eye blinking signal.

V. Jeyaramya et al. have proposed Mind controlled wheelchair using microcontroller that uses Microelectromechanical systems (MEMS) sensor to sense the direction of neck or hand to control the wheelchair in specified direction. Relay circuit is used to drive the wheels of the wheelchair, motor can be turned on and off using relay [8].

M. Akila et al. have designed low cost wheelchair based on Electromyography (EMG) technique which detects the muscle contractions in eye blinking. Single electrode is used to detect the signal which makes it cost efficient and less complex in terms of algorithm and interfaces. Using attention and meditation level by recording eye blink wheelchair can be controlled [9].

Nirmal T M has developed wheelchair that can be controlled by various ways, using joystick, Voice and eye movement. Multiple sensors are interfaced with microcontroller including temperature sensor, humidity sensor, obstacle detection sensor, PIR (Human Presence) sensor and heart beat analyzer. Motor driver with synchronous DC motor, sensors and controlling options interfaced with the microcontroller PIC16F877A and Arduino Mega, whereas wireless transceiver Xbee and GSM used [10].

D. Uma Mageswari et al. have developed wheelchair motion control guide using eye movement based on EEG signals, EOG electrodes are placed for capturing the signal and EEG signals to capture brain wave, these brain waves are controlled by microcontroller and transmitted using Zigbee module. Whereas DC motors are controlled by using relay, two relays are used which are controlled by switching transistors, by using both ON and OFF state of relays wheelchair will move forward and backwards [11]. Some of the commonly used process includes frequency classification in making Brain Computing interface (BCI), Nikhil R. Folane et al. have 
designed brain controlled wheelchair using ARM7 controller which receives command from MATLAB and generates respective interrupt signals [12].

\section{PROPOSED SYSTEM}

The wheelchair is controlled by acquiring signals from the brain and BCI (Brain Computing Interface) is used with the controller to perform the desired action by the patient as shown in Fig. 2. In any electric wheelchair configuration, the components like motors, batteries, controller, type of wheels plays important role in design and development of electric wheelchair. Selection of reliable wheels, using efficient motors and batteries, making the required controller in the wheelchair are important decision to take when designing and developing the electric wheelchair. As block diagram indicates that power supply distributed in all sections by two $12 \mathrm{v}$ batteries connected in series to two $12 \mathrm{v}$ motors and interfacing between $24 \mathrm{v}$ of battery and $5 \mathrm{v}$ of controller is implemented. Input is received from BCI by microcontroller and according to the signals output is generated through the motor driving IC that consists of Dual H-Bridge. This IC drive the motors according to the inputs received from BCI. Ultrasonic sensor module also interfaced with the microcontroller, The wheelchair can be controlled by the wheelchair controller that perform the functions available for control of speed variation and direction of the wheelchair, which is based on the output obtained from the signal processing. This is used to control the motors in real time wheelchair.

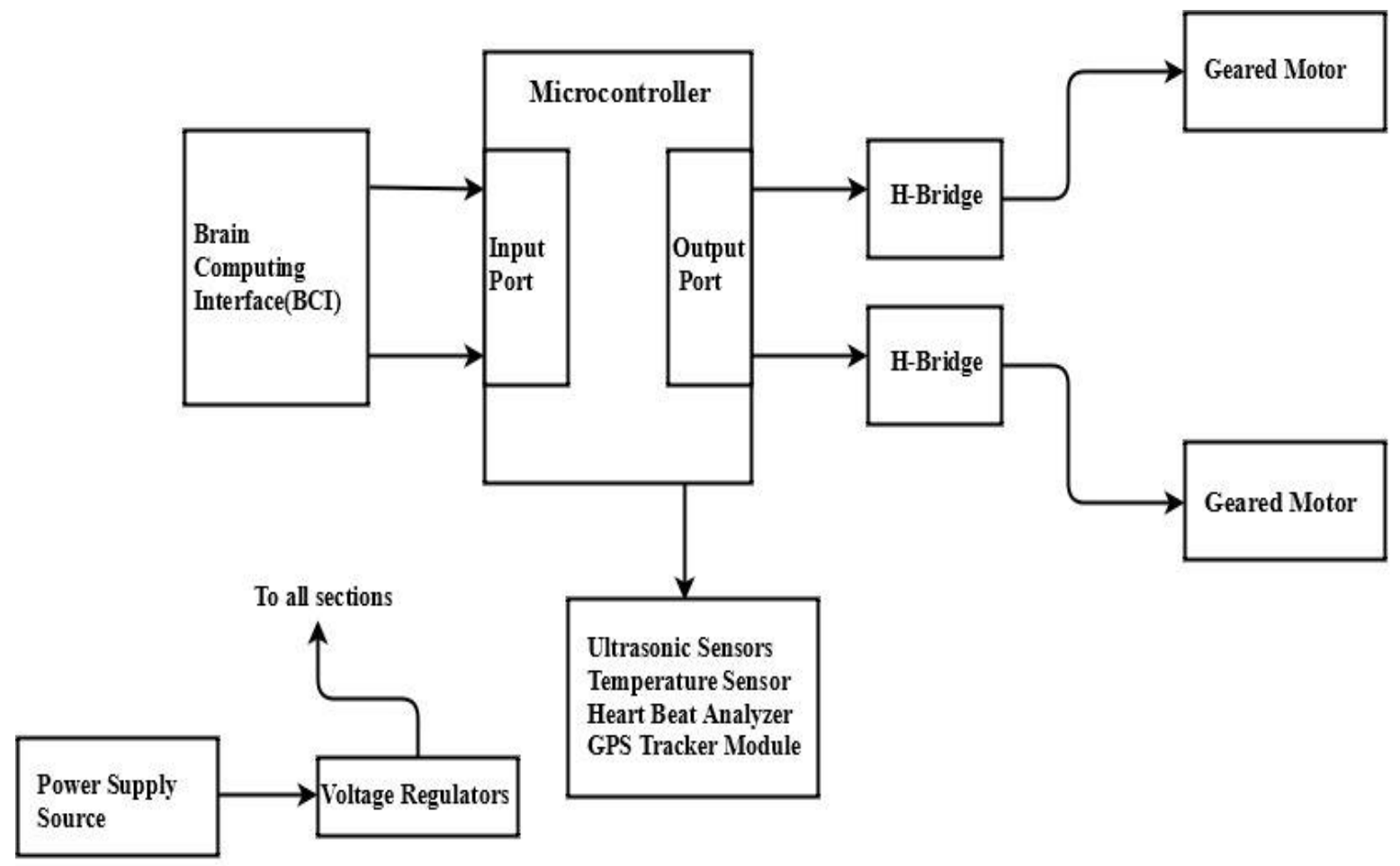

Fig. 2. Block diagram of proposed system

Motor Driver uses the dual $\mathrm{H}$ Bridge circuit that drive the two motors in bidirectional way, $\mathrm{H}$ Bridge is an electronic circuit that enables to provide the current in both the direction i.e. clockwise and anti-clockwise. These circuits are often used in many robots and small vehicle to allow DC motors to rotate forwards or backwards without causing much delay, some motor drivers are also available for single motor, these are also $\mathrm{H}$ Bridge but not dual H-bridge.

From brain computing interface, the signals are being received in form of serial data or some other pattern by the micro-control unit, different sensors can also be used with the microcontroller like temperature sensors, ultrasonic sensor etc. Ultrasonic sensors are used for obstacle detection 4 modules of sensor can easily be interfaced with microcontroller, these sensors are commonly available and also low price devices, one of the commonly ultrasonic sensor module is HCSR04 which is highly demanding in the field of robotics. Wheelchairs are comfortably driven using obstacle detecting ultrasonic sensor and also provides good efficiency in terms of power requirement. GPS module and various sensors like heart beat analyzer, temperature sensor can also be interfaced with the microcontroller. Additional features can be added with the wheelchair for providing the satisfactory control to the user and giving the required specification is most important thing, selecting efficient motors and batteries, clean and clear interfacing with motor driver to drive the motors easily, these factors play 
very important role. Ultrasonic sensors are mostly preferred due to low cost and perfect interfacing, Ultrasonic sensors working principle similar to SONAR, evaluate constitution of a target by interpreting the echoes from radio or sound waves, it produces high frequency waves and evaluate the echo which is received back by sensor. For continuous sensing, 4 set ultrasonic modules can be used.

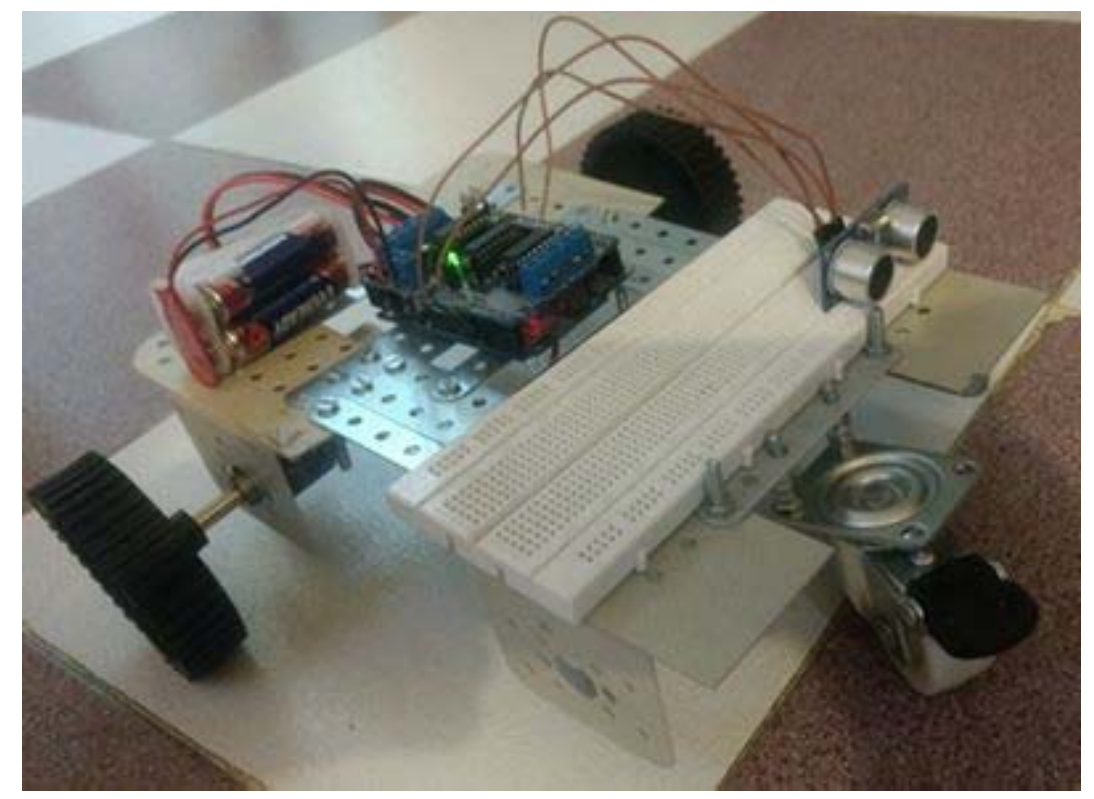

Fig. 3. Wheelchair Prototype developed

These modules can be tied to port of microcontroller to detect the obstacle in different way of the wheelchair, these sensor will provide trigger signal by activating trigger pin from microcontroller. Module will automatically transmit many ultrasonic burst and if there is an obstacle in front of ultrasonic module, it will reflect the ultrasonic burst. When the signal is back to the source, echo output of the sensor will be in high state for particular time duration taken for sending and receiving ultrasonic burst, pulse width has specific range which varies from 150 microseconds to 25 milliseconds. If no obstacle is detected the echo pulse will be about 38 milliseconds and motor will continue to rotate. We have discussed adding the features like dynamic suspension, fault alarm, calf support, tilt options etc. These features are most important if we consider the comfort zone of patient. Wheelchair prototype has been developed as shown in Fig. 3 which uses ultrasonic module connected with Arduino uno and serial data is received from brain computer interface by the microcontroller Arduino uno. This scale down model or prototype of the wheelchair uses geared motors because these motors are easily controllable using dual h-bridge motor driver or two separate h-bridge motor driver. Geared motor also consist of inbuilt gear box that uses gear reduction ratio which increases the torque by the same fraction it decreases the rotations per minute (RPM), these motors comes with high torque of $30 \mathrm{~kg}-\mathrm{cm}$ and current in these motors varies from $800 \mathrm{~mA}$ to $10 \mathrm{~A}$. Therefore, motor driver must be capable of providing $10 \mathrm{~A}$ per channel to have the highest rotational speed of the motor, other sensors like temperature sensor, hear beat analyzer, human presence sensor can also be interfaced with the same arduino that is used for driving motors but GPS module to be attached with another arduino to track the location of the user without any complexity in the hardware.

\section{CONCLUSION}

Design consideration of an EEG-based Brain-controlled wheelchair for elderly and disabled people has been discussed to lead their daily life without any difficulties. Methods are proposed to receive the signals from EEG processing used for making a motion with the direction of the movement and controlling the start/stop operations by using motor driver or relay braking system used with microcontroller. The performance based on various metrics and specifications analyzed, It can assist the people do their work without human interaction in moving their wheelchair and enrich their lifestyle. Though the BCI system is at its early development, but it requires improvement on the wheelchair and it should be more user-friendly. The wheelchair would be further enhanced by removing discomfort that sometimes lead to frustration and irritation in the user and focus on additional improvement of the features in wheelchair like obstacle detection, portability, providing dynamic suspension and the wheelchair can be controlled accurately without any collision. The proposed system can use efficient Bluetooth module by which signals can be transmitted to electric wheelchair control system, control signal transformed by MCU to control the DC motors in the electric wheelchair. 


\section{REFERENCES}

[1] Andrew M Kwarciak et al., "Evaluation of wheelchair tire rolling resistance using dynamometer-based coast-down tests," Journal of Rehabilitation Research and Development, vol. 46, no. 7, pp. 931-938, 2009.

[2] Tom Carlson and Jose del Millian, "Brain-controlled wheelchair: A Robotic Architecture," IEEE Robotics and Automation Magazine, vol. 20, no. 1, pp. 65-73, March 2013.

[3] N. Kobayashi and M. Nakagawa, "BCI-based control of electric wheelchair," 2015 IEEE 4th Global Conference on Consumer Electronics (GCCE), Osaka, pp. 429-430, 2015.

[4] A. Mirza et al., "Mind-controlled wheelchair using an EEG headset and arduino microcontroller," 2015 International Conference on Technologies for Sustainable Development (ICTSD), Mumbai, 2015, pp.1-5, 2015.

[5] D. W. K. Ng, Y. W. Soh and S. Y. Goh, "Development of an autonomous BCI Wheelchair," 2014 IEEE Symposium on Orlando, FL, 2014, pp. 1-4, 2014

[6] B. Jenita Amali Rani and A. Umamakeswari, "Electroencephalogram-besed brain controlled robotic wheelchair," Indian Journal of Science and Technology, vol. 8(S9), pp. 188-197, May 2015.

[7] Jzau-Sheng Lin and Win-Ching Yang, "Wireless brain-computer interface for electric wheelchairs with EEG and eye-blinking signals," International Journal of Innovative Computing Information and Control, vol. 8, no. 9, September 2012.

[8] Durgadevi, V. Jeyaramya and A. Asline Celes, "Mind controlled wheelchair using an EEG probes and microcontroller," International Journal for Innovative Research in Science \& Technology, vol. 2 pp. 240-243, 2016.

[9] M. Akila, K. SathiyaSekar and A. Suresh, "Smart brain controlled wheelchair and devices on EEG in low cost for disabled person," International Jourmal of Computers Communication Networks and Circuit Systems, vol. 1, Issue 1, April 2015.

[10] Nirmal T M, "Wheelchair for physically and mentally disabled persons," International Journal of Electrical and Electronics Research, vol. 2, pp. 112-118, June 2014.

[11] D. Uma Mageswari and G. Selvavinayagam, "Wheelchair motion control guide using eye movement based on EEG signals," International Journal of Engineering sciences and Research Technology, vol. 2, Issue 5, pp. 1122-1127, May 2013

[12] Nikhil R. Folane and R. M. Autee, "EEG based brain controlled wheelchair for physically challenged people," International Journal of Innovative Research in Computer and Communication Engineering, vol. 4, Issue 1, pp. 134-137, January 2016.

[13] http://www.1800wheelchair.com.

\section{AUTHOR PROFILE}

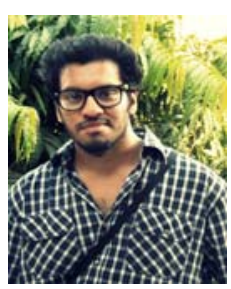

Ankit Kumar Mishra received Bachelor of Technology in Electronics and Communication Engineering in 2014 from Priyadarshini College of Computer Sciences, Greater Noida, India. $\mathrm{He}$ is currently pursuing Master of Engineering in VLSI and Embedded Systems from MIT College of Engineering, Pune, India. His research interest include VHDL, Verilog and Embedded Systems.

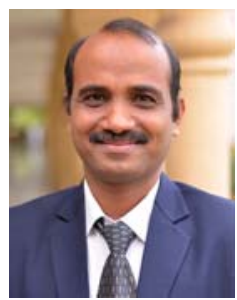

Krishna K. Warhade received the Bachelor of Engineering in Electronics in 1995 and Master of Engineering in Instrumentation in 1999 both from Shri Guru Govind Singhaji Institute of Engineering and Technology, Nanded, India and $\mathrm{PhD}$ in Nov 2010 from the department of Electrical Engineering, Indian Institute of Technology, Bombay, India. He has 21 years of teaching experience, he is currently working as a Dean R \& D at MIT College of Engineering, Pune, India. His research interest are in the area of Image Processing, Signal Processing, Video shot boundary detection, Wavelets and biomedical signal processing. 